\title{
Correction to: Understanding User Engagement Mechanisms on a Live Streaming Platform
}

Xinwei Wang and Dezhi Wu

\section{Correction to:}

Chapter "Understanding User Engagement Mechanisms on a Live Streaming Platform" in: F. F.-H. Nah and K. Siau (Eds.):

HCI in Business, Government and Organizations, LNCS 11589, https://doi.org/10.1007/978-3-030-22338-0_22

In the version of this chapter that was originally published, the word "conversion" was erroneously written as "conversation" in two places. This has now been corrected. 